\title{
On Schauder Frames in Conjugate Banach Spaces
}

\author{
S. K. Kaushik, ${ }^{1}$ S. K. Sharma, ${ }^{1}$ and Khole Timothy Poumai ${ }^{2}$ \\ ${ }^{1}$ Department of Mathematics, Kirori Mal College, University of Delhi, Delhi 110 007, India \\ ${ }^{2}$ Department of Mathematics, Motilal Nehru College, University of Delhi, Delhi 110 021, India
}

Correspondence should be addressed to S. K. Kaushik; shikk2003@yahoo.co.in

Received 31 August 2012; Accepted 17 November 2012

Academic Editor: Ding-Xuan Zhou

Copyright (C) 2013 S. K. Kaushik et al. This is an open access article distributed under the Creative Commons Attribution License, which permits unrestricted use, distribution, and reproduction in any medium, provided the original work is properly cited.

Weak* -Schauder frame in conjugate Banach spaces has been introduced and studied. A sufficient condition for the existence of weak ${ }^{*}$-Schauder frame in the conjugate space of a separable Banach space has been given. It has been shown that $\ell_{\infty}$ has weak ${ }^{*}-$ Schauder frame. Finally, a sufficient condition for the existence of a Schauder frame sequence has been given.

\section{Introduction}

Frames for Hilbert spaces were formally introduced by Duffin and Schaeffer [1]. Later, Daubechies et al. [2] found new applications to wavelets in which frames played an important role. Frames are main tools for use in signal and image processing, compression, sampling theory, optics, filter banks, signal detection, and so forth. In order to have more applications of frames, several notions generalizing the concept of frames have been introduced and studied, namely, pseudoframes [3], oblique frames [4], frames of subspaces (fusion frames) [5], G-frames [6], orthogonal frames [7, 8], and so forth.

Feichtinger and Gröchenig [9] extended the notion of atomic decomposition to Banach spaces. Gröchenig [10] introduced a more general concept for Banach spaces called Banach frame. Banach frames and atomic decompositions were further studied in [11-14]. Han and Larson [15] defined Schauder frame for a Banach space. In [16], Casazza, et al. gave various definitions of frames for Banach spaces including that of Schauder frame. In 2008, Casazza et al. [17] studied the coefficient quantization of Schauder frames in Banach spaces. Liu [18] gave the concepts of minimal and maximal associated bases with respect to Schauder frames and closely connected them with the duality theory. In [19], Liu and Zheng gave a characterization of Schauder frames which are near-Schauder bases. In fact, they generalized some results due to Holub [20]. Beanland et al. [21] proved that the upper and lower estimates theorems for finite dimensional decompositions of Banach spaces can be extended and modified to Schauder frames and gave a complete characterization of duality for Schauder frames. $\Phi$-Schauder frames were introduced and studied by Vashisht [22]. Recently, Liu [23] associated an operator with a Schauder frame and called it Hilbert-Schauder frame operator.

In the present paper, we introduce the concept of weak ${ }^{*}$ Schauder frame and weak-Schauder frame in conjugate Banach spaces. A sufficient condition for the existence of weak ${ }^{*}$-Schauder frame in a conjugate Banach space of a separable Banach space has been given. Also, an example of a conjugate space of a nonseparable Banach space which has no weak $^{*}$-Schauder frame is given. Further, it has been shown that $\ell_{\infty}$ has weak* ${ }^{*}$ Schauder frame. Finally, a sufficient condition for the existence of a Schauder frame sequence has been given.

\section{Preliminaries}

Throughout this paper, $E$ will denote a Banach space, $H$ will denote a Hilbert space, let $E^{*}$ the dual space of $E,\left[x_{n}\right]$ be the closed linear span of $\left\{x_{n}\right\}$ in the norm topology of $E$, and let $\pi$ be the canonical mapping of $E$ into $E^{* *}$. A series $\sum_{n=1}^{\infty} f_{i}$ in a conjugate Banach space $E^{*}$ is called weak-convergent to $f$ if it converges in $\sigma\left(E^{*}, E^{* *}\right)$-topology. In this case, we write $f \stackrel{w}{=} \sum_{n=1}^{\infty} f_{n}$. A series $\sum_{n=1}^{\infty} f_{i}$ in a conjugate Banach space $E^{*}$ is called weak ${ }^{*}$-convergent to $f$ if it converges in $\sigma\left(E^{*}, E\right)$ topology. In this case, we write $f \stackrel{w^{*}}{=} \sum_{n=1}^{\infty} f_{n}$. 
Definition 1. A sequence $\left\{x_{n}\right\}_{n \in \mathbb{N}} \subset H$ is called a frame for $H$ if there exist $A, B$ with $0<A \leq B<\infty$ such that

$$
A\|x\|^{2} \leq \sum_{n=1}^{\infty}\left|\left\langle x, x_{n}\right\rangle\right|^{2} \leq B\|x\|^{2}, \quad x \in H .
$$

The positive constants $A$ and $B$, respectively, are called lower and upper frame bounds of the frame $\left\{x_{n}\right\}_{n \in \mathbb{N}}$. The inequality (1) is called the frame inequality.

Definition 2. Let $E$ be a Banach space. A sequence $\left\{\left(x_{n}, f_{n}\right)\right\}_{n \in \mathbb{N}}\left(\left\{x_{n}\right\} \subset E,\left\{f_{n}\right\} \subset E^{*}\right)$ is called a Schauder frame for $E$ if

$$
x=\sum_{n=1}^{\infty} f_{n}(x) x_{n}, \quad x \in E .
$$

Definition 3. Let $E$ be a Banach space. A sequence $\left\{\left(x_{n}, f_{n}\right)\right\}_{n \in \mathbb{N}}\left(\left\{x_{n}\right\} \subset E,\left\{f_{n}\right\} \subset E^{*}\right)$ is called a Schauder frame sequence for $E$ if $\left\{\left(x_{n}, f_{n}\right)\right\}_{n \in \mathbb{N}}$ is a Schauder frame for $\left[x_{n}\right]$.

Next, we give the definition of a retro Banach frame introduced in [24].

Definition 4. Let $E$ be a Banach space and let $E^{*}$ be its conjugate space. Let $\left(E^{*}\right)_{d}$ be a Banach space of scalar-valued sequences associated with $E^{*}$, indexed by $\mathbb{N}$. Let $\left\{x_{n}\right\} \subset E$ and $T:\left(E^{*}\right)_{d} \rightarrow E^{*}$ be given. The pair $\left(\left\{x_{n}\right\}, T\right)$ is called a retro Banach frame for $E^{*}$ with respect to $\left(E^{*}\right)_{d}$ if

(i) $\left\{f\left(x_{n}\right)\right\} \in\left(E^{*}\right)_{d}, f \in E^{*}$,

(ii) there exist positive constants $A$ and $B$ with $0<A \leq$ $B<\infty$ such that

$$
A\|f\|_{E^{*}} \leq\left\|\left\{f\left(x_{n}\right)\right\}\right\|_{\left(E^{*}\right)_{d}} \leq B\|f\|_{E^{*}}, \quad f \in E^{*},
$$

(iii) $T$ is a bounded linear operator such that

$$
T\left(\left\{f\left(x_{n}\right)\right\}\right)=f, \quad f \in E^{*} .
$$

The positive constants $A$ and $B$, respectively, are called lower and upper frame bounds of the retro Banach frame $\left(\left\{x_{n}\right\}, T\right)$. The operator $T:\left(E^{*}\right)_{d} \rightarrow E^{*}$ is called the reconstruction operator (or the preframe operator). The inequality (3) is called the retro frame inequality. A retro Banach frame $\left(\left\{x_{n}\right\}, T\right)\left(\left\{x_{n}\right\} \subset E, T:\left(E^{*}\right)_{d} \rightarrow E^{*}\right)$ for $E$ with respect to $\left(E^{*}\right)_{d}$ with bounds $A, B$ is said to be tight, if it is possible to choose $A=B$, normalized tight, if $A=B=1$, and exact, if there exists no reconstruction operator $T_{0}$ such that $\left(\left\{x_{n}\right\}_{n \neq j}, T_{0}\right)(j \in \mathbb{N})$ is a retro Banach frame $E^{*}$.

Finally, we give the following results which will be used in the subsequent results.

Theorem 5 (see [25]). If $\left\{g_{n}\right\} \subset\left(\ell_{\infty}\right)^{*}$ and $\lim _{n \rightarrow \infty} g_{n}(x)=$ 0 , for all $x \in \ell_{\infty}$, then $\lim _{n \rightarrow \infty} \Phi\left(g_{n}\right)=0$ for all $\Phi \in E^{* *}$.

Theorem 6 (see [24]). Let $\left(\left\{x_{n}\right\}, T\right)\left(\left\{x_{n}\right\} \subset E, T:\left(E^{*}\right)_{d} \rightarrow\right.$ $\left.E^{*}\right)$ be a retro Banach frame for $E^{*}$ with respect to $\left(E^{*}\right)_{d}$. Then, $\left(\left\{x_{n}\right\}, T\right)$ is exact if and only if $x_{n} \notin\left[x_{i}\right]_{i \neq n}$, for all $n \in \mathbb{N}$.
In view of Theorem 6 , one may observe that if $\left(\left\{x_{n}\right\}, T\right)$ is an exact retro Banach frame for $E^{*}$, then there exists a sequence $\left\{g_{n}\right\}$ in $E^{*}$, called an admissible sequence to the retro Banach frame $\left(\left\{x_{n}\right\}, T\right)$, such that $g_{i}\left(x_{j}\right)=\delta_{i j}$, for all $i, j \in \mathbb{N}$.

\section{Main Results}

We begin with the following definitions of weak ${ }^{*}$-Schauder frame and weak-Schauder frame in $E^{*}$.

Definition 7. Let $E$ be a Banach space, let $\left\{f_{n}\right\}$ be a sequence in $E^{*}$ and let $\left\{\Phi_{n}\right\}$ be a sequence in $E^{* *}$, and let $M$ be a subset of $E^{*}$. Then, $\left\{\left(f_{n}, \Phi_{n}\right)\right\}_{n \in \mathbb{N}}$ is said to be

(a) weak-Schauder frame for $E^{*}$ with respect to $M$ if

$$
f \stackrel{w}{=} \sum_{n=1}^{\infty} \Phi_{n}(f) f_{n}, \quad f \in M
$$

(b) weak $^{*}$-Schauder frame for $E^{*}$ with respect to $M$ if

$$
f \stackrel{w^{*}}{=} \sum_{n=1}^{\infty} \Phi_{n}(f) f_{n}, \quad f \in M
$$

In particular, if $M=E^{*}$, then $\left\{\left(f_{n}, \Phi_{n}\right)\right\}_{n \in \mathbb{N}}$ is simply called a weak-Schauder frame and weak ${ }^{*}$-Schauder frame for $E^{*}$, respectively. Further, one may observe that if $\left\{\left(f_{n}, \Phi_{n}\right)\right\}_{n \in \mathbb{N}}$ is a Schauder frame for $E^{*}$, then it is also a weak ${ }^{*}$-Schauder frame (weak-Schauder frame) for $E^{*}$.

Now, we give an example of a conjugate space of a nonseparable Banach space which has no weak ${ }^{*}$-Schauder frame.

Example 8. $\ell_{\infty}^{*}$ has no weak $^{*}$-Schauder frame. Assume on the contrary that $\ell_{\infty}^{*}$ has a weak ${ }^{*}$-Schauder frame $\left\{\left(f_{n}, \Phi_{n}\right)\right\}_{n \in \mathbb{N}}\left(\left\{f_{n}\right\} \subset \ell_{\infty}^{*},\left\{\Phi_{n}\right\} \subset \ell_{\infty}^{* *}\right)$. Then, we have

$$
f(x)=\sum_{i=1}^{\infty} \Phi_{i}(f) f_{i}(x), \quad x \in E, f \in \ell_{\infty}^{*},
$$

that is,

$$
\left|f(x)-\sum_{i=1}^{n} \Phi_{i}(f) f_{i}(x)\right| \longrightarrow 0, \quad \text { as } n \longrightarrow \infty .
$$

So, by Theorem 5 , we have

$$
\lim _{n \rightarrow \infty} \Phi\left(f-\sum_{i=1}^{n} \Phi_{n}(f) f_{i}\right)=0, \quad \Phi \in \ell_{\infty}^{* *} .
$$

This gives $\ell_{\infty}^{*}$ is weak-separable. This is not possible. Hence, $\ell_{\infty}^{*}$ has no weak ${ }^{*}$-Schauder frame.

In view of Example 8 we have the following problem.

Problem 1. Does the conjugate space $E^{*}$ of a separable Banach space $E$ possess a weak ${ }^{*}$-Schauder frame? 
In this direction, we have the following result.

Proposition 9. If a Banach space E has a Schauder frame, then its conjugate Banach space has a weak ${ }^{*}$-Schauder frame.

Proof. Let $\left\{\left(x_{n}, f_{n}\right)\right\}_{n \in \mathbb{N}}$ be a Schauder frame for $E$; then

$$
x=\sum_{i=l}^{\infty} f_{i}(x) x_{i}, \quad x \in E .
$$

Therefore, for each $f \in E^{*}$, we have

$$
\begin{aligned}
f(x) & =f\left(\sum_{i=1}^{\infty} f_{n}(x) x_{n}\right) \\
& =\sum_{n=1}^{\infty} \pi\left(x_{n}\right)(f) f_{n}(x), \quad x \in E .
\end{aligned}
$$

Corollary 10. $\ell_{\infty}$ has weak ${ }^{*}$-Schauder frame.

Proof. It follows from Proposition 9.

Note. $\ell_{\infty}$ does not have a Schauder frame.

Towards the converse of Proposition 9, we have the following result.

Theorem 11. Let $E$ be a Banach space. Let $\left\{\left(f_{n}, \Phi_{n}\right)\right\}_{n \in \mathbb{N}}$ be a weak ${ }^{*}$-Schauder frame for $E^{*}$ such that each $\Phi_{n}$ is weak ${ }^{*}$ continuous. Then, there exists a sequence $\left\{x_{n}\right\} \subset E$ such that $\left\{\left(x_{n}, f_{n}\right)\right\}_{n \in \mathbb{N}}$ is a Schauder frame for $E$.

Proof. For each $f \in E^{*}$, we have

$$
\sum_{n=1}^{\infty} \Phi_{n}(f) f_{n} \stackrel{w^{*}}{=} f
$$

Since each $\Phi_{n}$ is weak ${ }^{*}$-continuous, there exists a sequence $\left\{x_{n}\right\} \subset E$ such that $\Phi_{n}=\pi\left(x_{n}\right), n \in \mathbb{N}$. So, we have

$$
\begin{aligned}
f(x) & =\sum_{n=1}^{\infty} \pi\left(x_{n}\right)(f) f_{n}(x) \\
& =f\left(\sum_{n=1}^{\infty} f_{n}(x) x_{n}\right), \quad x \in E, f \in E^{*} .
\end{aligned}
$$

This gives

$$
x=\sum_{n=1}^{\infty} f_{n}(x) x_{n}, \quad x \in E .
$$

In the following result, we characterize weak ${ }^{*}$-Schauder frame in terms of weak-Schauder frame with respect to a subset.

Theorem 12. Let $E$ be a Banach space. Let $\left\{f_{n}\right\}$ be a sequence in $E^{*}$ and let $\left\{\Phi_{n}\right\}$ be a sequence in $E^{* *}$. Then, $\left\{\left(f_{n}, \Phi_{n}\right)\right\}_{n \in \mathbb{N}}$ is a weak ${ }^{*}$-Schauder frame for $E^{*}$ if and only if $\left\{\left(f_{n}, \Phi_{n}\right)\right\}_{n \in \mathbb{N}}$ is a weak-Schauder frame for $E^{*}$ with respect to $\pi(E)$.
Proof. $\left\{\left(f_{n}, \Phi_{n}\right)\right\}_{n \in \mathbb{N}}$ is a weak ${ }^{*}$-Schauder frame for $E^{*}$ if and only if

$$
(\pi(x))(f)=\sum_{n=1}^{\infty} \Phi_{n}(f) \pi(x)\left(f_{n}\right), \quad f \in E^{*}, \pi(x) \in \pi(E)
$$

if and only if $\left\{\left(f_{n}, \Phi_{n}\right)\right\}_{n \in \mathbb{N}}$ is a weak-Schauder frame for $E^{*}$ with respect to $\pi(E)$.

Remark 13. Let $E$ be a reflexive Banach space. Let $\left\{f_{n}\right\}$ be a sequence in $E^{*}$ and $\left\{\Phi_{n}\right\}$ let be a sequence in $E^{* *}$. Then, by Theorem 12, $\left\{\left(f_{n}, \Phi_{n}\right)\right\}_{n \in \mathbb{N}}$ is a weak ${ }^{*}$-Schauder frame for $E^{*}$ if and only if $\left\{\left(f_{n}, \Phi_{n}\right)\right\}_{n \in \mathbb{N}}$ is a weak-Schauder frame for $E^{*}$.

Finally, we give a sufficient condition for the existence of a Schauder frame sequence in a Banach space.

Theorem 14. Let $E$ be a Banach space and let $\left(\left\{x_{n}\right\}, T\right)$ be an exact retro Banach frame for $E^{*}$ with admissible sequence $\left\{f_{n}\right\} \subset E^{*}$. Then, $\left\{\left(x_{n}, f_{n}\right)\right\}_{n \in \mathbb{N}}$ is a Schauder-frame sequence in $E$.

Proof. For each $n \in \mathbb{N}$, define $S_{n}: E \rightarrow E$ by

$$
S_{n}(x)=\sum_{i=1}^{n} f_{i}(x) x_{i}, \quad x \in E .
$$

Let $S_{n}^{*}$ be the adjoint operator to $S_{n}$. Then

$$
\begin{array}{r}
\left(S_{n}^{*}(g)\right)(x)=g\left(\sum_{i=1}^{n} f_{i}(x) x_{i}\right)=\left(\sum_{i=1}^{n} g\left(x_{i}\right) f_{i}\right)(x), \\
x \in E, \quad g \in E^{*}, \quad n=1,2,3 \ldots .
\end{array}
$$

This gives

$$
S_{n}^{*}(g)=\sum_{i=1}^{n} g\left(x_{i}\right) f_{i}, \quad g \in E^{*}, n=1,2,3, \ldots
$$

Thus, for every finite linear combination $g=\sum_{i=1}^{m} \beta_{i} f_{i}$, we have

$$
\begin{aligned}
S_{n}^{*}(g) & =\sum_{i=1}^{n} g\left(x_{i}\right) f_{i} \\
& =\sum_{i=1}^{m} \beta_{i} f_{i} \\
& =g, \quad n=m, m+1, \ldots .
\end{aligned}
$$

Let $f \in\left[f_{n}\right]$ and $\varepsilon>0$ be given. Then, there exists a finite linear combination $g=\sum_{j=1}^{m_{\varepsilon}} \beta_{j}^{(\varepsilon)} f_{j}$ such that $\|f-g\|<\varepsilon /(\nu+$ $1)$, where $v=\sup _{1 \leq n<\infty}\left\|s_{n}\right\|<\infty$. Also,

$$
\begin{aligned}
\left\|s_{n}^{*}(f)-f\right\| & \leq\left\|S_{n}^{*}(f)-S_{n}^{*}(g)\right\|+\left\|S_{n}^{*}(g)-g\right\|+\|g-f\| \\
& <\frac{v \varepsilon}{v+1}+\frac{\varepsilon}{v+1} \\
& =\varepsilon, \quad n=m_{\varepsilon}, m_{\varepsilon}+1, \ldots
\end{aligned}
$$




\section{Acknowledgment}

The authors thank the referee(s) for providing valuable comments and useful suggestions for the improvement of the paper.

\section{References}

[1] R. J. Duffin and A. C. Schaeffer, "A class of nonharmonic Fourier series," Transactions of the American Mathematical Society, vol. 72, pp. 341-366, 1952.

[2] I. Daubechies, A. Grossmann, and Y. Meyer, "Painless nonorthogonal expansions," Journal of Mathematical Physics, vol. 27, no. 5, pp. 1271-1283, 1986.

[3] S. Li and H. Ogawa, "Pseudo-duals of frames with applications," Applied and Computational Harmonic Analysis, vol. 11, no. 2, pp. 289-304, 2001.

[4] O. Christensen and Y. C. Eldar, "Oblique dual frames and shift-invariant spaces," Applied and Computational Harmonic Analysis, vol. 17, no. 1, pp. 48-68, 2004.

[5] P. G. Casazza and G. Kutyniok, "Frames of subspaces," in Wavelets, Frames and Operator Theory, vol. 345 of Contemporary Mathematics, pp. 87-113, American Mathematical Society, Providence, RI, USA, 2004.

[6] W. Sun, "G-frames and $g$-Riesz bases," Journal of Mathematical Analysis and Applications, vol. 322, no. 1, pp. 437-452, 2006.

[7] Virender, A. Zothansanga, and S. K. Kaushik, "On almost orthogonal frames," International Journal of Mathematics and Mathematical Sciences, vol. 2012, Article ID 920607, 6 pages, 2012.

[8] Z. Liu, G. Hu, and G. Wu, "Orthogonal multiwavelet frames in $L^{2}\left(R^{d}\right)$," Journal of Applied Mathematics, vol. 2012, Article ID 846852, 18 pages, 2012.

[9] H. G. Feichtinger and K. Gröchenig, "A unified approach to atomic decompositions via integrable group representations," in Proceedings of the Function Spaces and Applications, vol. 1302 of Lecture Notes in Mathematics, pp. 52-73, Springer, New York, NY, USA, 1988.

[10] K. Gröchenig, "Describing functions: atomic decompositions versus frames," Monatshefte für Mathematik, vol. 112, no. 1, pp. $1-42,1991$.

[11] O. Christensen and C. Heil, "Perturbations of Banach frames and atomic decompositions," Mathematische Nachrichten, vol. 185, pp. 33-47, 1997.

[12] P. K. Jain, S. K. Kaushik, and L. K. Vashisht, "On Banach frames," Indian Journal of Pure and Applied Mathematics, vol. 37, no. 5, pp. 265-272, 2006.

[13] S. K. Kaushik and S. K. Sharma, "On approximative atomic decompositions in Banach spaces," Communications in Mathematics and Applications, vol. 3, no. 3, pp. 293-301, 2012.

[14] S. K. Kaushik and S. K. Sharma, "On a generalization of atomic decompositions," Albanian Journal of Mathematics, vol. 5, no. 1, pp. 21-29, 2011.

[15] D. Han and D. R. Larson, Frames Bases and Group Representations, vol. 147 of Memoirs of the American Mathematical Society, 2000.

[16] P. G. Casazza, D. Han, and D. R. Larson, "Frames for Banach spaces," Contemporary Mathematics, vol. 247, pp. 149-182, 1999.

[17] P. G. Casazza, S. J. Dilworth, E. Odell, Th. Schlumprecht, and A. Zsák, "Coefficient quantization for frames in Banach spaces,"
Journal of Mathematical Analysis and Applications, vol. 348, no. 1, pp. 66-86, 2008.

[18] R. Liu, "On shrinking and boundedly complete Schauder frames of Banach spaces," Journal of Mathematical Analysis and Applications, vol. 365, no. 1, pp. 385-398, 2010.

[19] R. Liu and B. Zheng, "A characterization of Schauder frames which are near-Schauder bases," The Journal of Fourier Analysis and Applications, vol. 16, no. 5, pp. 791-803, 2010.

[20] J. R. Holub, "Pre-frame operators, Besselian frames, and nearRiesz bases in Hilbert spaces," Proceedings of the American Mathematical Society, vol. 122, no. 3, pp. 779-785, 1994.

[21] K. Beanland, D. Freeman, and R. Liu, "Upper and lower estimates for Schauder frames and atomic decompositions," In press, http://arxiv.org/abs/1202.2492v1.

[22] L. K. Vashisht, “On $\Phi$-Schauder frames," TWMS Journal of Applied and Engineering Mathematics, vol. 2, no. 1, pp. 116-120, 2012.

[23] R. Liu, "Hilbert-Schauder frame operators," Operators and Matrices, vol. 7, no. 1, pp. 91-99, 2013.

[24] P. K. Jain, S. K. Kaushik, and L. K. Vashisht, "Banach frames for conjugate Banach spaces," Zeitschrift für Analysis und ihre Anwendungen, vol. 23, no. 4, pp. 713-720, 2004.

[25] M. M. Day, Normed Linear Spaces, Springer, Berlin, Germany, 1958. 


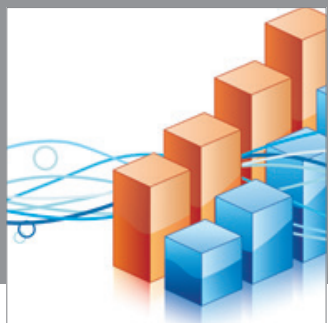

Advances in

Operations Research

mansans

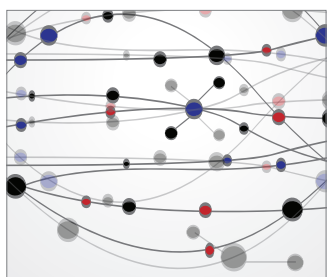

The Scientific World Journal
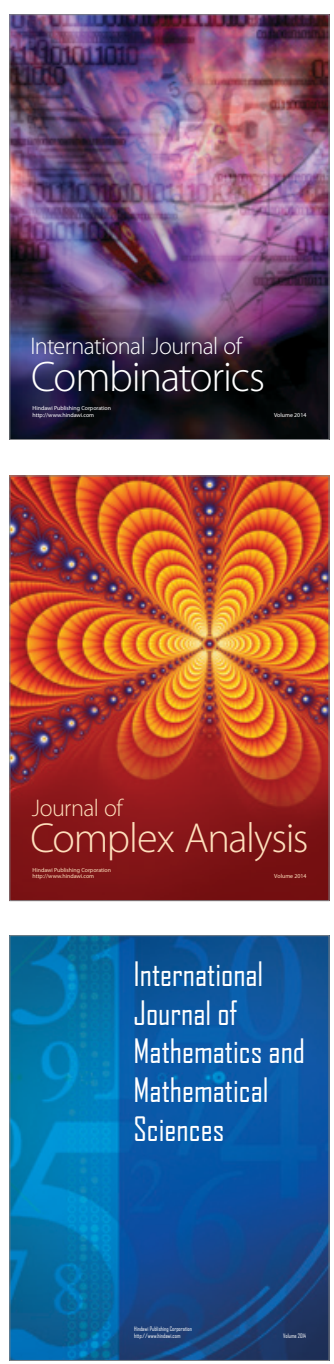
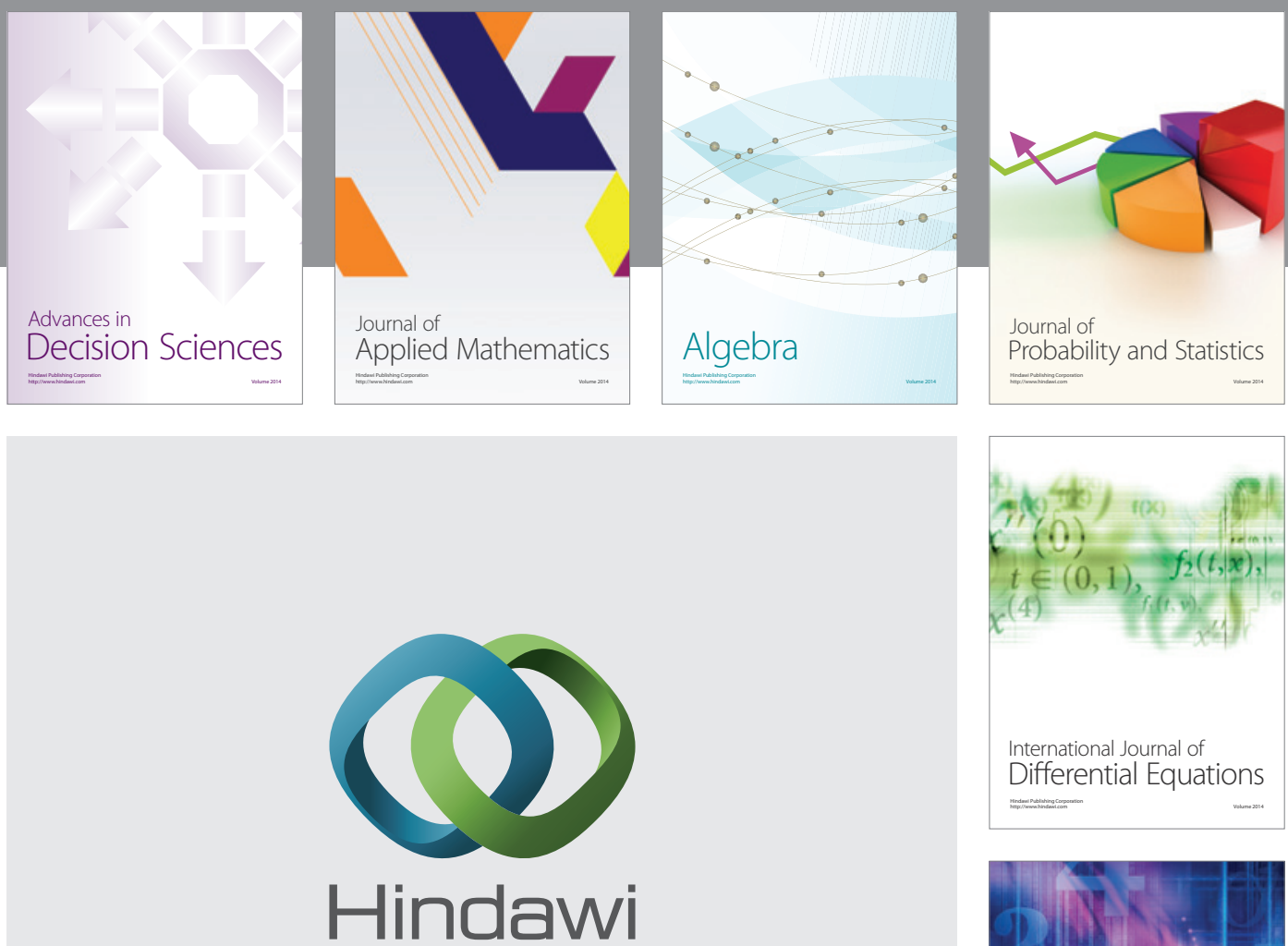

Submit your manuscripts at http://www.hindawi.com
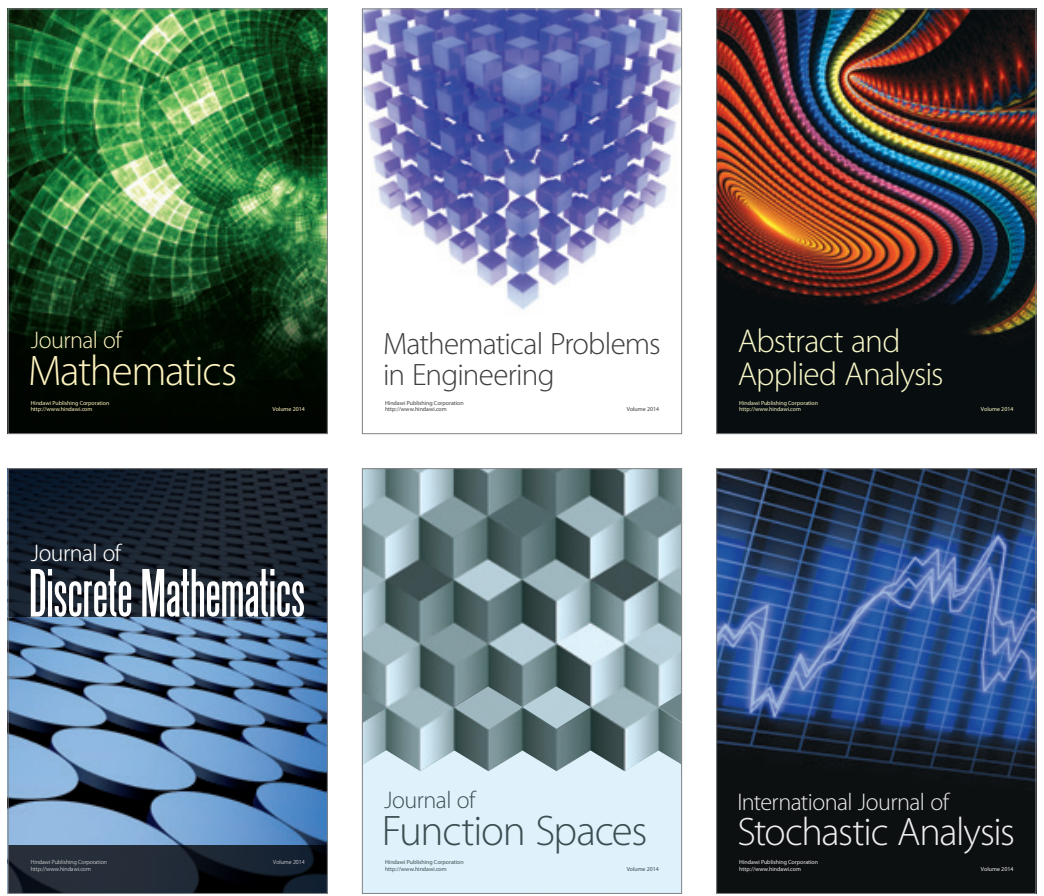

Journal of

Function Spaces

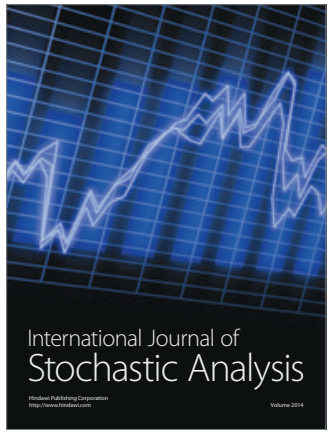

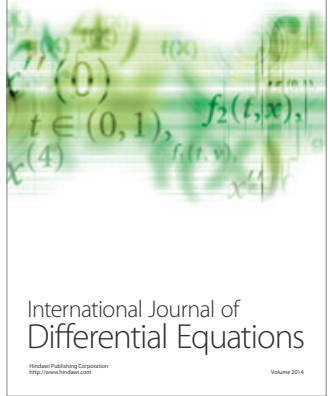
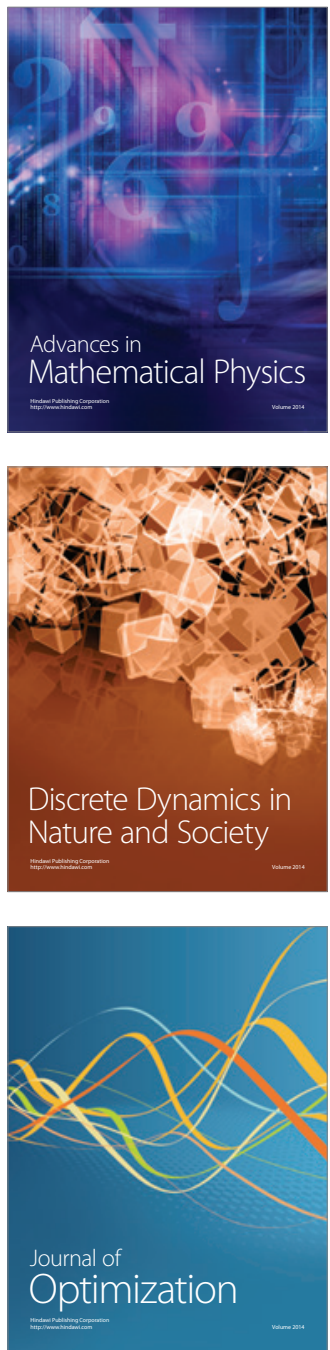